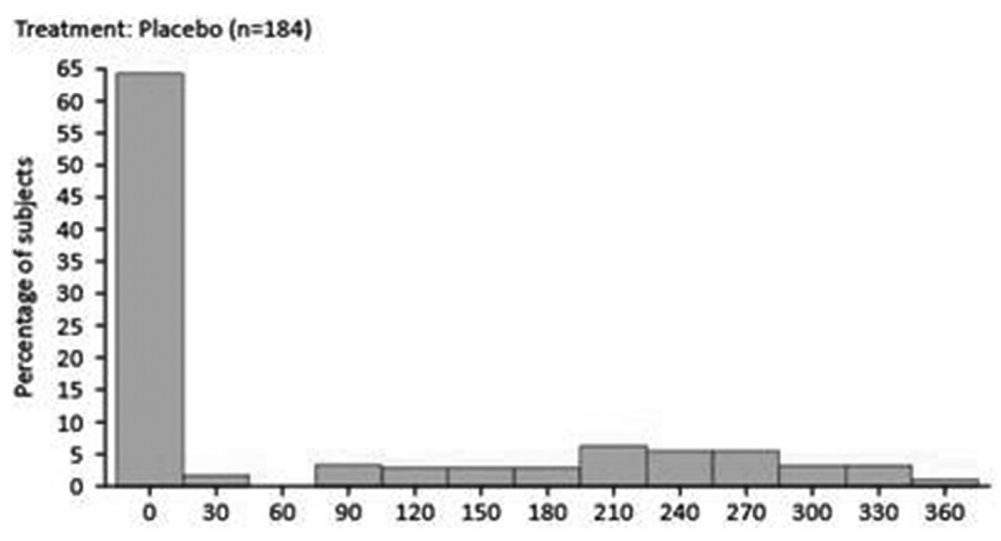

Number of days prednisone dose $57.5 \mathrm{mg} /$ day and/or reduced by $50 \%$ over baseline

Treatment: Belimumab $10 \mathrm{mg} / \mathrm{kg}(\mathrm{n}=352)$

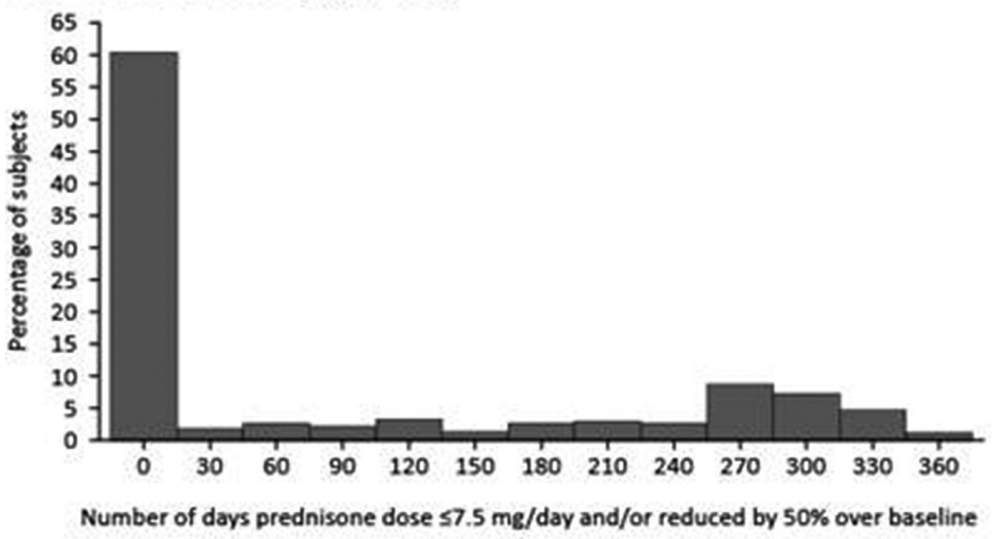

Abstract 106 Figure 1

(number of days $\leq 7.5 \mathrm{mg} / \mathrm{day}$ and/or dose reduced by $50 \%$ from baseline). The primary endpoint was SLE Responder Index response rate at Week 52 (reported elsewhere).

Results Baseline prednisone doses were similar between groups (Table 1). Across the population, cumulative prednisone dose over 52 weeks was significantly lower in the belimumab group versus placebo $(p=0.0005$; Table 1 . For subjects with baseline dose $>7.5 \mathrm{mg} /$ day, the median number of days that prednisone dose was $\leq 7.5 \mathrm{mg} / \mathrm{day}$ and/or reduced by $50 \%$ was zero in both groups; however, the $75^{\text {th }}$ percentile was larger for belimumab (213.5 days) versus placebo (172.0), reflecting the subjects who achieved longer durations of reduced steroid use within the belimumab group ( $p=0.0288$; Figure 1$)$. More subjects in the belimumab group had a dose reduction of $\geq 25 \%$ to $\leq 7.5 \mathrm{mg}$ /day during Weeks 40-52 (belimumab, 15.6\%; placebo, $10.9 \%$ : $p=0.0721)$. Adverse event incidences were similar (belimumab, 75.7\%; placebo, 74.9\%).

Conclusions These results suggest belimumab is more effective than placebo in reducing steroid use across 52 weeks in this population.

Study funded by GSK.

\section{7 \\ TARGETING SAHH AS A POTENTIAL THERAPEUTIC STRATEGY FOR AUTOIMMUNE DISEASES}

W Tang, Z Jianping*. Shanghai Institute of Materia Medica- Chinese Academy of Sciences, Immunopharmacology, Shanghai, China

\subsection{6/lupus-2017-000215.107}

Background and aims S-Adenosyl-1-homocysteine hydrolase $(\mathrm{SAHH})$ is a highly conserved ubiquitous enzyme that catalyses the hydrolysis of S-adenosyl-1-homocysteine (SAH) to adenosine (Ado) and homocysteine (Hcy) in eukaryotic cells. The reversible type III SAHH inhibitor DZ2002 has been found to have an immunomodulatory function and to alleviate disease in several inflammatory and autoimmune animal models, with greatly reduced cytotoxicity. This study was aim to examine the therapeutic effects and underlying mechanisms of DZ2002 on lupus-prone female NZB/W F1 mice.

Methods NZB/W F1 mice were treated orally with DZ2002 $(0.5 \mathrm{mg} \mathrm{kg}-1)$ for 11 weeks, and the proteinuria level and body weight were monitored. After the mice ware euthanized, serum biochemical parameters and renal damage were determined. Splenocytes of NZB/W F1 mice were isolated for ex 
vivo study. Toll-like receptor (TLR)-stimulated human PBMCs or murine bone marrow-derived dendritic cells were used for in vitro study.

Results Our study demonstrated that DZ2002 exerted a therapeutic effect on NZB/W F1 mice with established nephritis. The mechanism involves the modulation of $\mathrm{T}$ cell development in lupus by interfering with TLR-triggered APC function. Further study explored the regulatory mechanisms of SAHH on DC function in both innate and adaptive immune system, using the SAHH inhibitor with definite target and potent immunosuppressive activity.

Conclusions The present issue demostrated that the reversible SAHH inhibitor DZ2002 effectively ameliorates lupus syndrome in NZB/W F1 mice by regulating TLR signaling-mediated APC responses. This compound is prospective to become a novel drug for SLE treatment with Hcy as a potential biomarker in autoimmune disease.

\section{IGURATIMOD INHIBITS HUMAN B CELL TERMINAL DIFFERENTIATION IN VITRO AND MAY BENEFIT PATIENTS WITH REFRACTORY LUPUS NEPHRITIS}

${ }^{1} \mathrm{Q}$ Yan*, ${ }^{1} \mathrm{Y} Y \mathrm{Ye},{ }^{2} \mathrm{X}$ Zhang, ${ }^{1} \mathrm{C}$ Bao. ${ }^{1}$ RenJi Hospital- School of Medicine- Shanghai JiaoTong University, Rheumatology, Shanghai, China; ${ }^{2}$ Chinese Academy of Sciences, Institute Pasteur of Shanghai, Shanghai, China

\subsection{6/lupus-2017-000215.108}

Background and Aims Iguratimod (IGT) is a small molecular immunomodulatory drug and has been approved for treating rheumatoid arthritis. In our previous work, IGT ameliorates lupus-like disease in MRL/lpr mice by inhibiting abnormal B cell differentiation. The aim of this study is to further investigate the effects of IGT on human B cells.

Methods We established a set of stimulations to induce naive human $\mathrm{B}$ cell into plasmablast $(\mathrm{PB})$ in vivo. We also enrolled 7 patients with refractory lupus nephritis (LN) to assess the potential efficacy of IGT.

Results IGT significantly attenuates the generation of CD19 +CD20-CD27hiCD38hi plasma cells upon both BCR-dependent and independent stimulations. IGT affects neither proliferation or apoptosis of B cell in vitro. In further investigation on B cell differentiation signalling pathwasys, we identifies that Blimp-1 and Xbp-1 can be remarkably impaired by IGT both in transcriptional and protein level; while Jak/STAT or NF-кB signalling are intact with IGT treatment. For explosive clinical study, seven patients with refractory LN were enrolled and administrated with IGT and steroids. ALL of these patients surprisingly show improved proteinuria after 8 week treatment. One patient quit the treatment because of anaemia.

Conclusions IGT has a unique effect to arrest B cell terminal differentiation, which provides strong evidence that this drug could be a new candidate drug to treat B cell related autoimmune diseases such as lupus.

\section{LONG-TERM DATA ON SIROLIMUS TREATMENT IN} LUPUS NEPHRITIS PATIENTS

DYH Yap, MK Ma, MM Mok, LP Kwan, GC Chan, TM Chan. Queen Mary Hospital- The University of Hong Kong, Department of Medicine, Hong Kong, Hong Kong S.A.R
Background and aims Preliminary data suggested efficacy of sirolimus in treatment of lupus nephritis (LN), but its longterm efficacy and tolerability data is lacking.

Methods We reviewed Class III/IV/V LN patients who received prednisolone and sirolimus either as initial or maintenance treatment during Jan 2007 to Jan 2016.

Results Sixteen patients were included (duration of sirolimus treatment: $27.2 \pm 19.6$ months). Ten patients received sirolimus due to intolerance to standard immunosuppressive treatments and six patients because of a history of malignancy. Five patients received sirolimus during active $\mathrm{LN}$, and showed improvement in proteinuria $(2.8 \pm 1.9 \mathrm{~g} /$ day and $0.1 \pm 0.1 \mathrm{~g} /$ day at baseline and 36 months, $\mathrm{p}=0.011$ compared to baseline), anti-dsDNA $(107.7 \pm 91.9 \mathrm{IU} / \mathrm{mL}$ and $37.0 \pm 55.4 \mathrm{IU} / \mathrm{mL}$ at baseline and 36 months, $\mathrm{p}=0.145)$ and C3 $(54.8 \pm 26.1 \mathrm{mg} / \mathrm{dL}$ and $86.3 \pm 18.6 \mathrm{mg} / \mathrm{dL}$ at baseline and 36 months, $\mathrm{p}=0.084$ ). Eleven patients received sirolimus during disease quiescence, and showed significant improvement in C3 $(90.4 \pm 18.1 \mathrm{mg} / \mathrm{dL}$ and $117.7 \pm 25.1 \mathrm{mg} / \mathrm{dL}$ at baseline and 36 months, $\mathrm{p}=0.025$ ) and stable renal function $(58.5 \pm 25.2 \mathrm{ml} / \mathrm{min}$ and 56.7 $\pm 29.0 \mathrm{~mL} / \mathrm{min}$ at baseline and 36 months, $\mathrm{p}=0.199)$ and proteinuria $(0.8 \pm 0.7 \mathrm{~g} / \mathrm{day}$ and $0.7 \pm 0.7 \mathrm{~g} / \mathrm{day}$ at baseline and 36 months, $p=0.263)$. One patient, whose serum creatinine was $244 \mu \mathrm{mol} / \mathrm{L}$ when sirolimus was started, developed renal failure after 27 months. Renal flare occurred in one patient after 36 months. Sirolimus was discontinued in five patients including one with leucopenia. Four patients showed lipid profile deterioration which was adequately controlled with statin.

Conclusions Sirolimus can be an alternative treatment option for $\mathrm{LN}$ and the long-term results do not suggest excessive adverse effects.

\section{SUCCESSFUL TREATMENT OF REFRACTORY LUPUS NEPHRITIS WITH SECUKINUMAB IN A PATIENT COMPLICATED WITH PSORIASIS VULGARIS}

H Yoshinari* ${ }^{*}$ K Nakano, S Nakayamada, S Iwata, S Kubo, I Miyagawa, M Yoshikawa, Y Miyazaki, Y Satoh, K Saito, Y Tanaka. University of Occupational and Environmental Health, The first department of internal medicine, Kitakyushu, Japan

\subsection{6/lupus-2017-000215.110}

Background and aims We report the case of a 62-year-old woman. Psoriasis Vulgaris (Psoriasis) was diagnosed in X-31 year and also SLE with nephritis (WHO IIIA) in X-11. She was treated with high-dose methylprednisolone and cyclosporine A (CsA) to achieve remission. Methylprednisolone was reduced to $4 \mathrm{mg} /$ day.

Methods Because of renal dysfunction, although CsA was discontinued in May X, psoriasis, renal dysfunction and proteinuria became further worse, she was admitted to hospital in July X. She was diagnosed with SLE flare with class IV-G(A/ $\mathrm{C})+\mathrm{V}$ lupus nephritis (INS/RPS) and associated psoriasis. The SLEDAI score was 16 and psoriasis area and severity index (PASI) score was 16. Although high-dose corticosteroid (1 mg/ $\mathrm{kg} /$ day) and a concomitant first dose of IV cyclophosphamide (IVCY) were started, anasarca was still observed and S-Cr was increased from 1.98 to $2.85 \mathrm{mg} / \mathrm{dL}$. Because proportion of activated Th17 cells were increased in peripheral blood(PD), and the infiltration of many lymphocytes and IL-17-positive cells in renal interstitium, secukinumab, an antibody against IL-17A, was administered. 